ARTICLE

\title{
Investigation and management of primary immunodeficiency in South African children
}

\author{
B Eley, ${ }^{1} \mathrm{MB} \mathrm{ChB}, \mathrm{FCPaed}, \mathrm{BSc}$ (Hons); M Esser, ${ }^{2} \mathrm{MB} \mathrm{ChB}, \mathrm{MMed}$ Paed (Cert Rheum) \\ ${ }^{1}$ Paediatric Infectious Diseases Unit, Red Cross War Memorial Children's Hospital and Department of Paediatrics and Child Health, \\ University of Cape Town, South Africa \\ ${ }^{2}$ Immunology Unit, National Health Laboratory Service, Tygerberg, and Department of Pathology, Tygerberg Hospital and Stellenbosch University, \\ Cape Town, South Africa
}

Corresponding author: B Eley (brian.eley@uct.ac.za)

\begin{abstract}
The primary immunodeficiency diseases (PIDs) are inherited, non-communicable diseases that cause immunological dysfunction. PIDs are seldom reported in South Africa (SA). Based on a mid-2013 population estimate of 52.98 million and assuming that the prevalence of PIDs is similar to that in well-resourced settings, the total number of individuals with PIDs in our country should range between 2850 and 45 723. However, fewer than 500 cases of PID have been reported in SA. Between five and 15 new, fully characterised PIDs are reported annually. Our understanding of the physiology of the immune system has been substantially enhanced by these discoveries, and consequently the international classification of PIDs has been updated.
\end{abstract}

S Afr Med J 2014;104(11):793. DOI:10.7196/SAMJ.8946

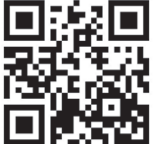

The primary immunodeficiency diseases (PIDs) are inherited, non-communicable diseases that cause immunological dysfunction. They primarily manifest with a wide range of infections. The pattern, frequency and severity of infection depend on the underlying immunological defect (Table 1), and geographical and social determinants. For example, in high tuberculosis (TB) prevalence settings chronic granulomatous disease (CGD) may manifest with recurrent TB.

In well-resourced countries, estimates of the prevalence of PIDs vary widely from $5.38 / 100000$ in France to $86.3 / 100000$ in the USA. ${ }^{[1,2]}$ There is very little reporting of PIDs from Africa, including South Africa (SA). In the latest Jeffrey Modell Foundation (JMF) global survey, 1463 patients with PIDs out of a global total of 77193 were from Africa. ${ }^{[3]}$ This low reporting prevalence of $1.9 \%$ contrasts with Africa's contribution of approximately $15 \%$ to the global population. Recent speculation suggests that as many as 902000 PID cases should be reported from Africa. ${ }^{[1]}$ In SA, based on a mid-2013 population estimate of 52.98 million and assuming that the prevalence of PIDs is similar to that in well-resourced settings, the total number of people with PIDs should range between 2850 and 45723 . However, fewer than 500 PID cases have been reported in SA. ${ }^{[4,5]}$

The almost 300 patients in the SA National PID Registry reflect deficiencies similar to those in European and US data, with the majority due to antibody deficiencies. Respiratory infections are the most common presenting symptom and a positive family history is recorded in $30 \%$. Paediatricians outside of tertiary care centres contribute a significant third of registry entries. Of special concern is the under-reporting of PIDs from disadvantaged communities. As infections are common in childhood, a high level of awareness is needed to investigate those that may be caused by an immune deficit.

\section{Classification of PIDs}

Between five and 15 new, fully characterised PIDs are reported annually. Our understanding of the physiology of the immune system has been substantially enhanced by these discoveries, and consequently the international classification of PIDs has been updated. It now includes nearly 250 different PIDs spanning nine categories, including a new category, phenocopies of PIDs, which contains conditions caused by somatic mutations and autoantibodymediated PIDs (Table 2). ${ }^{[6]}$ Given the complexity of the human immune system, the rate at which new PIDs are being discovered will most likely continue for the foreseeable future, necessitating further revision to the international classification.

\section{Investigation}

The medical history, clinical presentation and causative organism guide the laboratory investigation of PIDs.

Red flag signs in the medical history include severe, persistent, unusual and recurrent (SPUR) infections and, very importantly, a history of previous infant death due to infections or a known family history of PID. The frequency of infection commonly indicates the need for further investigations. Six or more upper respiratory infections per year are acceptable in the average toddler. ${ }^{[7]}$ Increased frequency is seen with overcrowding and poor sanitation. Aggravating factors for recurrent infections such as allergy or daycare have to be evaluated. Secondary immunodeficiencies such as HIV infection are far more common and have to be excluded initially. Repeated infections at the same site indicate a possible anatomical cause.

The clinical presentation of PIDs may include features of autoimmunity or inflammation, apart from those of infections. The JMF warning signs (Table 3) provide a useful guide to the need for investigation, but in the SA context have to include bacillus Calmette-Guérin (BCG) dissemination, recurrent meningococcal infections and awareness of infections with atypical mycobacteria. The following warning signs were found to be most predictive of PID in children: a family history of PID, use of intravenous antibiotics to treat sepsis and failure to thrive in T-lymphocyte PID. ${ }^{[8]}$ 
Table 1. Spectrum of infections

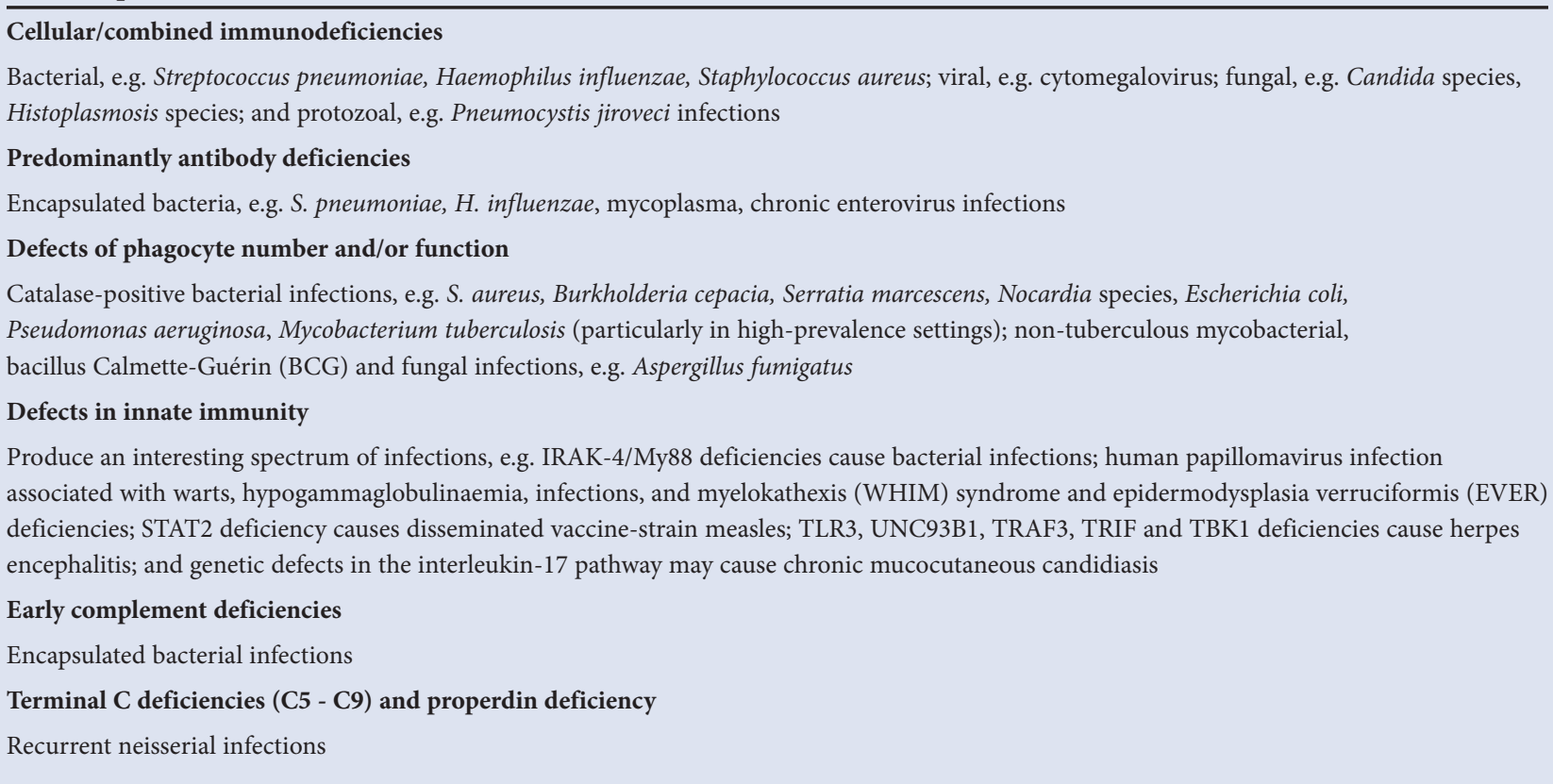

Table 2. Major categories of primary immunodeficiency diseases (PIDs)

- Combined (T- and B-cell) immunodeficiencies

- Combined immunodeficiencies with associated or syndromic features

- Predominantly antibody deficiencies

- Diseases of immune dysregulation

- Defects of phagocytic number, function or both

- Defects in innate immunity

- Autoinflammatory disorders

- Complement deficiencies

- Phenocopies of PIDs

Table 3. Ten warning signs of primary immunodeficiency developed by the Jeffrey Modell Foundation Medical Advisory Board $^{[16]}$

- Four or more new ear infections within one year

- Two or more serious sinus infections within one year

- Two or more months of antibiotics, with little effect

- Two or more pneumonias within one year

- Failure of an infant to gain weight or grow normally

- Recurrent, deep skin or organ abscesses

- Persistent thrush in mouth or fungal infection on skin

- Need for intravenous antibiotics to clear infections

- Two or more deep-seated infections including septicaemia

- A family history of primary immunodeficiency

The causative organisms may be defined as virulent, opportunistic, hospital or community acquired and assist in determining the relevant immunodeficiency to be investigated: deficiency for intracellular (e.g. TB) versus extracellular bacterial targeted immune responses. Infections with encapsulated organisms may point to a deficient immune response specific to Streptococcus pneumoniae, with antibody responses being crucial. T-cell PIDs, although commonly manifesting with intracellular infections, generally also involve B-cell-dependent (humoral) immune responses and hence result in bacterial infections. T-cell-related immune deficit manifests early in life, sometimes in the first months, whereas B-cell PIDs tend to present with symptoms at the time of loss of maternal passive antibodies in the infant, especially $>6$ months of age.

Basic laboratory screening (full blood count, differential count, immunoglobulins and complement) is readily available to identify the majority of significant defects. A more comprehensive four-step laboratory approach has been developed by the JMF and modified for use in SA.

Step 1. After exclusion of HIV and, where indicated on history, an assessment for atopy, the investigation starts with a full blood count and, importantly, differential count for neutrophil and lymphocyte numbers. A persistently low lymphocyte count for age, which should be $>1 / 3$ of leukocytes $<3$ years of age or $>3.0 \times 10^{\%} / \mathrm{L}$, is an important indicator of possible severe combined immunodeficiency (SCID) or combined immunodeficiencies. A blood smear will assist, e.g. to identify small platelets as seen in Wiskott-Aldrich syndrome. Serum immunoglobulin (Ig) G, M, A and E, total complement activity and subsequent fractions as indicated by the absence of activity, cystic fibrosis screening and investigation for active TB where suspected on history and clinical examination complete step 1.

Step 2. Vaccine antibody response to a range of antigens but more targeted to polysaccharide-specific antigens (pneumococcus) and protein antigens (tetanus) is indicated with recurrent bacterial infections, even in the presence of normal immunoglobulin levels. Patients have to be off immunoglobulin replacement therapy for 6 months. These responses should be repeated four weeks after vaccine boosting to determine an appropriate increase in IgG, of which there are four subclasses. These can be further investigated for deficiency of one or more, especially if total IgG levels are normal in the presence of 
recurrent infections. Both IgG subclass deficiency and deficient vaccine antibody response, especially to $S$. pneumoniae, should be investigated concurrent with symptomatic IgA deficiency ( $\operatorname{IgA}<0.08 \mathrm{~g} / \mathrm{L}$ ), as these are common associations.

Lymphocyte subsets are enumerated by flow cytometry with cluster of differentiation (CD) markers to document B-cell numbers (CD19), which are absent in X-linked agammaglobulinaemia when all immunoglobulin isotypes are severely reduced. Total T cells (CD3) are measured with the sum of T-helper (CD4) and T-suppressor (CD8) cells, and natural killer cells are measured as CD16 and CD56.

Step 3. Lymphocyte enumeration does not provide information on function, which can be done at specialised laboratories on request. The response to mitogens, e.g. phytohaemagglutinin, or to antigens, e.g. Candida, can give information on specific immune deficit or suppression. Neutrophil function, particularly in suspected deficiency such as CGD, can be evaluated with the neutrophil burst activity. This test, done on the mother in X-linked CGD, can also guide on possible carrier status. Neutrophil studies for leukocyte adhesion, chemotaxis and phagocytosis, lymphocyte subset definitions of naïve and memory status are all requested after specialist consultation.

Stage 4. Cytokine studies, enzyme studies and genetic molecular investigations are not generally available and can only be selectively done.

While neonatal screening for SCIDs is becoming routine practice in North America and Europe by T-cell-receptor excision circle assay, this is costly and only available on request in SA. Dissemination of routinely applied neonatal BCG vaccine remains an important alerting sign outside HIV infection. ${ }^{[9]}$

\section{Management}

Most interventions for managing PIDs are available to a varying degree in SA.

\section{Immunisation practice}

Immunisation with live viral or bacterial vaccines poses an infection risk to individuals with severe PIDs of T-cell, B-cell and phagocytic origin, requiring modification to routine immunisation practice of children with PIDs and changes to the immunisation of household contacts of patients with PIDs. For example, recent evidence suggests that $51 \%$ of infants with SCID experience BCG-related complications following neonatal immunisation, 34\% develop BCGosis and 17\% develop localised BCG complications. ${ }^{[10]}$ Oral polio and smallpox vaccines are contraindicated in children with severe antibody deficiencies. For children with combined B- and T-cell deficiencies, such as SCID, all live vaccines are contraindicated, and for those with phagocytic disorders live bacterial vaccines are contraindicated. ${ }^{[11]}$

Immunocompetent household contacts of PID patients should not receive smallpox or oral polio vaccine, because these vaccine virus strains may be transmitted to immunocompromised patients. Other vaccines may be administered to close contacts because they pose little risk of transmission to the PID patient. ${ }^{[1]}$

\section{Antimicrobial prophylaxis}

Several PIDs may benefit from antimicrobial prophylaxis. In milder antibody conditions, such as IgA deficiency or transient hypogammaglobulinaemia of infancy, recurrent infection may occasionally require short courses of prophylactic antibiotics. Antibiotic prophylaxis should be the first intervention for prevention in patients with specific antibody deficiency or IgG subclass deficiency and recurrent infection, before considering immunoglobulin replacement therapy (IRT). Antibiotic prophylaxis may be added in patients with severe antibody deficiency if IRT is unable to adequately prevent recurrent infections. Both antibacterial and antifungal prophylactic agents are required in children with severe phagocytic disorders. Prior to haematopoietic stem cell transplantation (HSCT), children with severe combined immunodeficiencies such as SCID require broad-spectrum antibacterial, antiviral and antifungal prophylaxis. Detailed prophylactic regimens have recently been published. ${ }^{[12]}$

\section{Immunoglobulin replacement therapy}

Many predominantly antibody deficiencies and combined immunodeficiencies in which quantitative and/or qualitative IgG deficiency occurs may benefit from IRT. Lifelong IRT is required for severe, genetically confirmed deficiencies in which arrest in early B-cell development is demonstrated, such as X-linked agammaglobulinaemia. Incompletely defined predominantly antibody deficiencies characterised by the presence of circulating $\mathrm{B}$ cells and subnormal IgG concentration may require a 12-month trial of IRT, while documenting the clinical response. If the response is favourable, treatment may be extended for $1-5$ years, followed by periodic re-evaluation of serum immunoglobulin concentrations as hypogammaglobulinaemia may be transient and resolve spontaneously. Functional antibody responses should ideally not be checked until IRT has been discontinued for at least 6 months. If IgG deficiency persists, IRT should be restarted and may have to be continued indefinitely if the deficiency fails to resolve. Treatment with intravenous immunoglobulin is most widely used. Subcutaneous immunoglobulin administration is less frequently used but is equally effective. Intravenous immunoglobulin is administered at 3 - 4-week intervals at the usual starting dose of $400 \mathrm{mg} / \mathrm{kg}$ for patients without bronchiectasis and $600 \mathrm{mg} / \mathrm{kg}$ for those with bronchiectasis. After achieving steady state, an IgG trough concentration of $>5 \mathrm{~g} / \mathrm{L}$ reduces the infection rate and trough levels up to $10 \mathrm{~g} / \mathrm{L}$ will progressively reduce the risk of pneumonia. Progressive adjustment of the total infusion dose is required to optimise the IgG trough concentration. ${ }^{[13]}$

\section{Additional measures to control infection}

General advice aimed at reducing the risk of infection will benefit many immunodeficient patients, including advice on personal, hand and dental hygiene, avoidance of exposure to individuals with active infections and annual influenza immunisation for household contacts. Antimicrobial prophylaxis is often administered with other interventions to reduce the risk of infection. In SCID, prior to an HSCT, the infant should ideally be managed in a laminar flow facility, with full contact precautions, and receive sterile feeds. In the immediate post-HSCT period, similar management interventions apply. In CGD, life-long interferon- $\gamma$ is recommended in combination with co-trimoxazole and itraconazole prophylaxis. However, the high cost precludes its routine use in SA. In Job syndrome, which causes a markedly elevated IgE concentration, the risk of colonisation and infection with Staphylococcus aureus may be reduced by regular bleach or chlorhexidine soaks and intermittent mupirocin applications.

\section{Haematopoietic stem cell transplantation}

This is the treatment of choice for many severe PIDs, including SCID. In SA, there is limited access to HSCT in the public sector. However, three children with SCID have been successfully transplanted, permitting the development of guidelines for the pre-HSCT management of children with SCID, including the tissue typing procedure and identification of suitable donors. ${ }^{[14]}$ 


\section{Other therapies}

Other therapeutic modalities include cytokine therapy, such as myeloid haematopoietic growth factors for congenital and cyclical neutropenia, enzyme replacement for adenosine deaminase deficiency and C1-inhibitor deficiency, colchicine in familial Mediterranean fever (FMF), and interleukin 1-blocking agents in autoinflammatory disorders including patients with colchicine-resistant FMF. Gene therapy offers hope as a potential treatment and cure for PIDs in the future. ${ }^{[15]}$

\section{Conclusion}

PIDs worldwide are more common than is generally believed.$^{[1]}$ In SA, diagnosis may be masked by a high background prevalence of infectious diseases. It is hoped that this article increases awareness of PIDs among general practitioners and paediatricians.

\section{References}

1. Bousfiha AA, Jeddane L, Ailal F, et al. Primary immunodeficiency diseases worldwide: More common than generally thought. J Clin Immunol 2013;33(1):1-7. [http://dx.doi.org/10.1007/s10875-012-9751-7] Boyle JM, Buckley RH. Population prevalence of diagnosed primary immunodeficiency diseases in the United States. J Clin Immunol 2007;27(5):497-502.

3. Modell V, Knaus M, Modell F, Roifman C, Orange J, Notarangelo LD. Global overview of primary mmunodeficiencies: A report from Jeffrey Modell Centers worldwide focused on diagnosis, treatment and discovery. Immunol Res 2014;60(1):132-144. [http://dx.doi.org/10.1007/s12026-014-8498-z] 4. Naidoo R, Ungerer L, Cooper M, Pienaar S, Eley BS. Primary immunodeficiencies: A 27-year review at
a tertiary paediatric hospital in Cape Town, South Africa. J Clin Immunol 2011;31(1):99-105. [http:// dx.doi.org/10.1007/s10875-010-9465-7]

5. Esser M. Primary immunodeficiency: Missed opportunities and treatment challenges. Curr Allergy Clin Immunol 2012;25:184-188.
6. Al-Herz W, Bousfiha A, Casanova JL, et al. Primary immunodeficiency diseases: An update on the classification from the international union of immunological societies expert committee for primary .

7. Gray PE, Namasivayam M, Ziegler JB. Recurrent infection in children: When and how to investigate for primary immunodeficiency? J Paediatr Child Health 2012;48(3):202-209. [http://dx.doi.org/10.1111/ j.

Subbarayan A, Colarusso G, Hughes SM, et al. Clinical features that identify children with primary immunodeficiency diseases. Pediatrics 2011;127(5):810-816. [http://dx.doi.org/10.1542/peds.2010-3680] . Hesseling AC, Johnson LF, Jaspan H, et al. Disseminated bacille Calmette-Guérin disease in HIV infected South African infants. Bull World Health Organ 2009;87(7):505-51

10. Marciano BE, Huang $\mathrm{C}-\mathrm{Y}$, Joshi $\mathrm{G}$, et al. $\mathrm{BCG}$ vaccination in patients with severe combined immunodeficiency Complications, risks, and vaccination policies. J Allergy Clin Immunol 2014;133(4):1134-1141. [http://dx.doi.

org/10.1016/j.jaci.2014.02.028]
11. Medical Advisory Committee of the Immune Deficiency Foundation, Shearer WT, Fleisher TA, et al. Recommendations for live viral and bacterial vaccines in immunodeficienct patients and their close relatives. J Allergy Clin Immunol 2014;133(4):961-966. [http://dx.doi.org/10.1016/j.jaci.2013.11.043]

12. Papadopoulou-Alataki E, Hassan A, Davies EG. Prevention of infection in children and adolescen with primary immunodeficiency disorders. Asian Pac J Allergy Immunol 2012;30(4):249-258

13. Orange JS, Grossman WJ, Navickis RJ, Wilkes MM. Impact of trough IgG on pneumonia incidence in primary immunodeficiency: A meta-analysis of clinical studies. Clin Immunol 2010;137(1):21-30. [http://dx.doi.org/10.1016/j.clim.2010.06.012]

14. Eley B, Hendricks M, Davidson A, Lochan H, Schlaphoff T, Maart S. Primary immunodeficiency disease (PIDs): Diagnostic and treatment considerations for patients managed at Red Cross War Memorial Children's Hospital (RCWMCH), August 2014. http://www.paediatrics.uct.ac.za/sites/default/files/image_tool/ images $/ 38 / 1.5 .10 \% 20 \mathrm{PIDs}$-diagnostic\% $20 \% 20$ treatment $\% 20$ considerations.pdf (accessed 20 August 2014).

15. Zhang L, Thrasher AJ, Gaspar HB. Current progress on gene therapy for primary immunodeficiencies. Gene Ther 2013;20(10):963-969. [http://dx.doi.org/10.1038/gt.2013.21]

16. Jeffrey Modell Foundation Medical Advisory Board. Ten warning signs of primary immunodeficiency. http://www.info4pi.org (accessed 23 September 2014).

\section{Useful information sources}

- African Society for Immunodeficiency Diseases (ASID): http://www.asid.m

- Immune Deficiency Foundation (IDF): http://www.primaryimmune.org

- International Patient Organization for Primary Immunodeficiency (IPOPI): http:// www.ipopi.org

- Primary Immunodeficiency Network South Africa (PiNSA): http://www.pinsa.org

Primary Immunodeficiency Resource Center: http://www.info4pi.or 